\title{
Acesso e qualidade da atenção secundária e da assistência em estomatologia no Brasil
}

I ${ }^{1}$ Rhuan Vitor Sodré Leal, ${ }^{2}$ Danielle Tupinambá Emmi,

${ }^{3}$ Marizeli Viana De Aragão Araújo I

Resumo: Objetivo: analisar a assistência especializada em saúde bucal nos Centros de Especialidades

Odontológicas (CEO) do Brasil, com ênfase em

Estomatologia, tendo em vista o aumento de novos casos de câncer de boca no país. Metodologia: análise de dados secundários do $1^{\circ}$ ciclo do Programa de Melhoria do Acesso e Qualidade dos CEO (PMAQ-CEO), do Instituto Brasileiro de Geografia e Estatística (IBGE) e Departamento de Atenção Básica do Ministério da Saúde (DAB-MS). Resultados: somente 13,98\% dos municípios brasileiros são cobertos por $\mathrm{CEO}$, sendo a Estomatologia uma das especialidades mais deficientes, presente em somente $65,55 \%$ dos CEO participantes do PMAQ-CEO. A realização da biópsia ocorre em 82,3\% dos CEO, mas em $60 \%$ dos serviços resultado do exame ainda é demorado e o suprimento de recursos materiais é insuficiente. Conclusão: o retrato da Estomatologia no Brasil compromete o acesso ao diagnóstico, tratamento e contribui para afecçóes e morbidades bucais, como o câncer bucal.

> Palavras-chave: Avaliação de serviços de saúde; Atenção Secundária à Saúde; Saúde bucal; Câncer bucal.

\author{
1 Universidade Federal do Pará. \\ Belém-PA, Brasil (rhuanvitorsleal@ \\ gmail.com). \\ ORCID: 0000-0002-1028-7725 \\ ${ }^{2}$ Universidade Federal do Pará. \\ Belém-PA, Brasil (dtemmi@ufpa.br). \\ ORCID: 0000-0002-6046-0717 \\ ${ }^{3}$ Universidade Federal do Pará. \\ Belém-PA, Brasil (marizeli.araujo@ \\ gmail.com). \\ ORCID: 0000-0002-9103-9263
}

Recebido em: 14/05/2020

Aprovado em: 08/01/2021

Revisado em: 24/05/2021 


\section{Introdução}

A mudança da assistência odontológica centrada na doença e em práticas mutiladoras para um modelo de atenção integral à saúde ainda é um desafio constante nos serviços de saúde. No âmbito da saúde pública brasileira, essa mudança foi possível em decorrência da implementação da Política Nacional de Saúde Bucal (PNSB) - Brasil Sorridente pelo Ministério da Saúde (MS), em 2004 que impactou positivamente na ampliação da assistência odontológica e, consequentemente, na condição de saúde bucal do brasileiro (PUCCA JUNIOR, 2013).

Dentre as diretrizes da PNSB, pode-se destacar a reorganização da atenção básica em saúde bucal, com a ampliação das Equipes de Saúde Bucal (ESB) na Estratégia Saúde da Família (ESF), bem como a ampliação e qualificação da atenção secundária, por meio da implantação dos Centros de Especialidades Odontológicas (CEO) e dos Laboratórios Regionais de Próteses Dentárias (LRPD). Como parte da ampliação e qualificação da atenção, encontram-se os processos do trabalho em saúde, de gestáo, de educação permanente/continuada e de avaliação de serviços (BRASIL, 2000; BRASIL 2004a).

Os CEO representaram uma inovação desta política como atenção secundária em saúde bucal, integrando a rede hierarquizada do Sistema Único de Saúde (SUS), sendo referência à atenção primária. Este serviço oferta, no mínimo, as especialidades de Endodontia, Periodontia, Pacientes com Necessidades Especiais, Cirurgia Oral Menor e Diagnóstico Bucal (Estomatologia), com ênfase no diagnóstico e detecção do câncer de boca.

Apesar da melhora da assistência e da condição de saúde bucal da população, dados do Instituto Nacional de Câncer (INCA) evidenciam que a incidência do câncer bucal no Brasil é considerada uma das mais altas do mundo, sendo o quinto mais incidente em homens e o décimo terceiro em mulheres. Somente em 2017, 4.923 homens e 1.372 mulheres vieram a óbito vitimados por câncer na cavidade oral e para cada ano do triênio 2020-2022, estimam-se 11.180 casos novos de câncer da cavidade oral em homens e de 4.010 em mulheres no país (INCA, 2019).

Desse modo, é evidente que o acesso à especialidade de Estomatologia é fundamental no sistema público de saúde brasileiro. Entretanto, o Brasil é um país de dimensôes continentais e os determinantes e condicionantes da saúde, bem como os serviços de saúde, não são equânimes nas macrorregiôes brasileiras (PUCCA JÚNIOR, 2006; 
IBGE, 2010; SALIBA et al., 2013; BALDANI et al., 2018). Sendo assim, diante da diversidade e desigualdades no contexto social e do sistema de saúde brasileiro, há a necessidade de saber se o brasileiro está tendo acesso aos serviços de saúde e se a assistência é de qualidade, o que somente é possível por meio da avaliação dos serviços oferecidos à população, como uma prática rotineira e continuada nos diversos setores da saúde para o diagnóstico do serviço, objetivando que o acesso e os padróes adequados de qualidade sejam equivalentes nos diferentes municípios, estados e regiôes do País.

Nesse cenário, foi instituído no âmbito da PNSB, por meio da Portaria no 261/ GM/MS de 21 de fevereiro de 2013, o Programa Nacional de Melhoria do Acesso e da Qualidade dos Centros de Especialidades Odontológicas (PMAQ-CEO), que visa por meio da indução de processos, o constante monitoramento dos serviços, buscando assegurar maior acesso e qualidade à população. O $1^{\circ}$ ciclo do PMAQCEO aconteceu em 2014 e foi organizado em ciclos complementares e contínuos de 4 fases: 1) adesão e contratualização; 2) desenvolvimento; 3) avaliação externa e certificação; 4) recontratualização (BRASIL, 2013).

A primeira e segunda fase do PMAQ-CEO compreendem a pactuação de compromissos entre as gestôes municipais e estaduais com o Ministério da Saúde (MS). Para estimular a participação dos CEO no PMAQ, o Ministério da Saúde disponibilizou a parcela de $20 \%$ do incentivo financeiro denominado de Componente de Qualidade da Atenção Especializada em Saúde Bucal, que consiste no valor repassado ao Município ou Estado no qual o serviço que aderiu ao programa está vinculado.

Já na $3^{\text {a }}$ fase - Avaliação Externa, os CEO são avaliados quanto à infraestrutura, recursos materiais, recursos humanos, desempenho dos profissionais, a realização da educação permanente dos profissionais atuantes, que consiste na constante qualificação do profissional, cumprimento do o apoio matricial e cooperação horizontal, que consiste no compartilhamento de conhecimento e informaçôes entre as equipes profissionais da atenção básica e especializada, para a resolubilidade e integralidade da atenção à saúde, além da gestão e satisfação do usuário.

É na $3^{\text {a }}$ fase que também são avaliados se os CEO realizam a autoavaliação e o monitoramento de indicadores para o estabelecimento de metas que melhorem o acesso e qualidade dos serviços prestados aos usuários. A partir desses critérios, os quais compreendem a avaliação externa, autoavaliação e monitoramento de indicadores, os CEO são certificados quanto ao seu desempenho. 
Ressalta-se que na fase de avaliação externa, o levantamento de informaçóes sobre a estrutura do CEO, organização do serviço e processo de trabalho, bem como, a percepção e satisfação do usuário, fornecem subsídios e informaçôes importantes sobre a adequação aos padróes de qualidade recomendados pelo Ministério da Saúde, assim como, por meio da autoavaliação, o reconhecimento das fragilidades do serviço, fomentando o aprimoramento de estratégias e a indução de mudanças que visem à qualificação da atenção e o maior acesso e qualidade dos serviços.

Desse modo, o objetivo deste estudo é analisar a situação da assistência especializada em saúde bucal nos Centros de Especialidades Odontológicas (CEO) do Brasil, com ênfase em Estomatologia, tendo em vista esta ser uma das especialidades obrigatórias no CEO e o considerável impacto à saúde pública, em decorrência das morbidades e óbitos resultantes da falta de acesso a este serviço.

\section{Métodos}

Este é um estudo descritivo, quantitativo, de caráter avaliativo, que utilizou dados secundários coletados na fase da Avaliação Externa do $1^{\circ}$ ciclo do PMAQCEO, ocorrido em 2014. Para o diagnóstico dos serviços de atenção secundária, foi realizada a correspondência dos dados coletados do PMAQ-CEO, com dados referentes à implantação e cobertura dos Centros de Especialidades Odontológicas disponíveis pelo Instituto Brasileiro de Geografia e Estatística (IBGE) e Sala de Apoio a Gestão Estratégica (SAGE) do Departamento de Atenção Básica (DAB) do Ministério da Saúde (IBGE, 2013; PMAQ-CEO, 2014; SAGE, 2019)

Em todo o Brasil 932 CEO distribuídos em 26 estados e no Distrito Federal participaram da avaliação externa do PMAQ-CEO, trazendo importantes indicadores para analisar a atenção especializada em saúde bucal. A avaliação externa foi estruturada em 3 módulos, sendo o módulo 1 representado pela observação direta, avaliando questóes relacionadas à infraestrutura do CEO, materiais, insumos, equipamentos e medicamentos; o módulo 2 constituído pela entrevista com profissionais (gestores e cirurgiôes dentistas dos CEO) para obtenção de informaçôes acerca do processo de trabalho; e o módulo 3 relacionado à percepção e a satisfação dos usuários sobre o acesso e uso dos serviços ofertados.

Neste estudo foram analisados apenas os módulos 1 e 2, por serem os que consideravam várias dimensões e subdimensóes do serviço em Estomatologia, 
gerando diferentes indicadores como microdados. Os dados foram analisados de forma descritiva com frequências calculadas utilizando-se o programa Microsoft Office Excel Professional Plus número 365, versão do ano de 2016.

Consonante à Resolução no 466 de 12 de dezembro de 2012 do Conselho Nacional de Saúde e Resolução no 510 de 7 de abril de 2016 da Comissão Nacional de Ética em Pesquisa (CONEP) do Conselho Nacional de Saúde (CNS) este estudo não necessita de aprovação pelo Comitê de Ética em Pesquisa, por tratar-se de informaçóes de domínio público.

\section{Resultados}

Dos 988 CEO implantados no Brasilem 2013, 932 (94,33\%) serviços participaram do $1^{\circ}$ Ciclo do PMAQ-CEO, de acordo com o censo realizado. A participação no programa ocorreu de forma satisfatória em todo o país, sendo a menor média na macrorregiáo centro-oeste, com cerca de $88,57 \%$ de CEO participantes, conforme a Tabela 1.

Tabela 1. Frequências absoluta (N) e relativa (\%) da cobertura da Atençẫo Secundária no Brasil e participaçáo dos CEO no 1 Ciclo do PMAQ- CEO, em 2013.

\begin{tabular}{cccccc}
\hline $\begin{array}{c}\text { Regióes } \\
\text { e } \\
\text { Estados }\end{array}$ & $\begin{array}{c}\text { CEO } \\
\text { implantados } \\
\text { no Brasil de } \\
\text { acordo com o } \\
\text { DAB }\end{array}$ & $\begin{array}{c}\text { CEO } \\
\text { participantes } \\
\text { do PMAQ } \\
\mathbf{N}\left(\%^{a}\right.\end{array}$ & $\begin{array}{c}\text { Número de } \\
\text { municípios } \\
\text { por UF } \\
\text { (IBGE) }\end{array}$ & $\begin{array}{c}\text { Municípios } \\
\text { que possuem } \\
\text { cobertura } \\
\text { de CEO } \\
\text { participantes } \\
\text { do PMAQ } \\
\text { N (\%) }\end{array}$ & $\begin{array}{c}\text { Total de CEO } \\
\text { participantes } \\
\text { do PMAQ } \\
\text { N (\%) }\end{array}$ \\
\hline Regiáo Norte & $\mathbf{6 6}$ & $\mathbf{6 0}\left(\mathbf{9 0 , 9 ^ { a } )}\right.$ & $\mathbf{4 5 0}$ & $\mathbf{4 7}(\mathbf{1 0 , 4 4 )}$ & $\mathbf{6 0}(\mathbf{6 , 5})$ \\
\hline RO & 9 & $7\left(77,7^{a}\right)$ & 52 & $5(9,61)$ & $7\left(11,66^{c}\right)$ \\
AC & 3 & $2\left(60,6^{a}\right)$ & 22 & $1(4,54)$ & $2\left(3,33^{c}\right)$ \\
AM & 11 & $11\left(100^{a}\right)$ & 62 & $9(14,51)$ & $11\left(18,33^{c}\right)$ \\
RR & 1 & $1\left(100^{a}\right)$ & 15 & $1(6,66)$ & $1\left(1,66^{c}\right)$ \\
PA & 32 & $29\left(90,6^{a}\right)$ & 144 & $22(15,27)$ & $29\left(48,33^{c}\right)$ \\
AP & 3 & $3\left(100^{a}\right)$ & 16 & $2(12,5)$ & $3\left(5^{c}\right)$ \\
TO & 7 & $7\left(100^{a}\right)$ & 139 & $7(5,03)$ & $7\left(11,66^{c}\right)$ \\
\hline
\end{tabular}




\begin{tabular}{|c|c|c|c|c|c|}
\hline $\begin{array}{l}\text { Regióes } \\
\text { e } \\
\text { Estados }\end{array}$ & $\begin{array}{c}\text { CEO } \\
\text { implantados } \\
\text { no Brasil de } \\
\text { acordo com o } \\
\text { DAB }\end{array}$ & $\begin{array}{c}\text { CEO } \\
\text { participantes } \\
\text { do PMAQ } \\
\mathrm{N}(\%)^{a}\end{array}$ & $\begin{array}{l}\text { Número de } \\
\text { municípios } \\
\text { por UF } \\
\text { (IBGE) }\end{array}$ & $\begin{array}{c}\text { Municípios } \\
\text { que possuem } \\
\text { cobertura } \\
\text { de CEO } \\
\text { participantes } \\
\text { do PMAQ } \\
\text { N (\%) }\end{array}$ & $\begin{array}{c}\text { Total de CEO } \\
\text { participantes } \\
\text { do PMAQ } \\
\text { N (\%) }\end{array}$ \\
\hline $\begin{array}{c}\text { Regiáo } \\
\text { Nordeste }\end{array}$ & 383 & $357\left(93,21^{a}\right)$ & 1.794 & $320(17,83)$ & $357\left(38.4^{b}\right)$ \\
\hline MA & 28 & $26\left(92,85^{a}\right)$ & 217 & $23(10,59)$ & $26\left(7,28^{c}\right)$ \\
\hline PI & 28 & $28\left(100^{a}\right)$ & 224 & $27(12,05)$ & $28\left(7,84^{c}\right)$ \\
\hline CE & 88 & $80\left(90,9^{a}\right)$ & 184 & $66(35,86)$ & $80\left(22,40^{c}\right)$ \\
\hline RN & 24 & $23\left(95,83^{a}\right)$ & 167 & $20(11,97)$ & $23\left(6,44^{c}\right)$ \\
\hline $\mathrm{PB}$ & 56 & $53\left(94,64^{a}\right)$ & 223 & $49(21,97)$ & $53\left(9,51^{c}\right)$ \\
\hline $\mathrm{PE}$ & 48 & $40\left(83,33^{a}\right)$ & 185 & $36(19,45)$ & $40\left(7,18^{c}\right)$ \\
\hline $\mathrm{AL}$ & 22 & $22\left(100^{a}\right)$ & 102 & $21(20,58)$ & $22\left(6,16^{c}\right)$ \\
\hline SE & 10 & $10\left(100^{a}\right)$ & 75 & $10(13,33)$ & $10\left(2,80^{c}\right)$ \\
\hline BA & 79 & $75\left(94,93^{a}\right)$ & 417 & $68(16,30)$ & $75\left(21,01^{c}\right)$ \\
\hline $\begin{array}{l}\text { Regiáo } \\
\text { Sudeste }\end{array}$ & 351 & $337\left(96,01^{a}\right)$ & 1.668 & $271(16,24)$ & $337\left(36.2^{b}\right)$ \\
\hline MG & 85 & $83\left(97,64^{a}\right)$ & 853 & $77(9,02)$ & $83\left(24,62^{c}\right)$ \\
\hline ES & 10 & $9\left(90^{a}\right)$ & 78 & $8(10,25)$ & $9\left(2,67^{c}\right)$ \\
\hline RJ & 68 & $66\left(97,05^{a}\right)$ & 92 & $42(45,65)$ & $66\left(19,58^{c}\right)$ \\
\hline SP & 188 & $179\left(95,21^{a}\right)$ & 645 & $144(22,32)$ & $179\left(53,11^{c}\right)$ \\
\hline Região Sul & 118 & $116\left(98,3^{a}\right)$ & 1.191 & $96(8,06)$ & $116\left(12.5^{b}\right)$ \\
\hline PR & 50 & $48\left(96^{a}\right)$ & 399 & $37(9,27)$ & $48(41,37)$ \\
\hline SC & 43 & $43\left(100^{a}\right)$ & 295 & $38(12,88)$ & $43\left(37,06^{c}\right)$ \\
\hline RS & 25 & $25\left(100^{a}\right)$ & 497 & $21(4,22)$ & $25\left(21,55^{c}\right)$ \\
\hline
\end{tabular}




\begin{tabular}{cccccc}
\hline $\begin{array}{c}\text { Regióes } \\
\text { e } \\
\text { Estados }\end{array}$ & $\begin{array}{c}\text { CEO } \\
\text { implantados } \\
\text { no Brasil de } \\
\text { acordo com o } \\
\text { DAB }\end{array}$ & $\begin{array}{c}\text { CEO } \\
\text { participantes } \\
\text { do PMAQ } \\
\mathbf{N}(\%)^{a}\end{array}$ & $\begin{array}{c}\text { Número de } \\
\text { municípios } \\
\text { por UF } \\
\text { (IBGE) }\end{array}$ & $\begin{array}{c}\text { Municípios } \\
\text { que possuem } \\
\text { cobertura } \\
\text { de CEO } \\
\text { participantes } \\
\text { do PMAQ } \\
\text { N (\%) }\end{array}$ & $\begin{array}{c}\text { Total de CEO } \\
\text { participantes } \\
\text { do PMAQ } \\
\text { N (\%) }\end{array}$ \\
\hline $\begin{array}{c}\text { Regiáo } \\
\text { Centro-Oeste }\end{array}$ & $\mathbf{7 0}$ & $\mathbf{6 2 ( 8 8 , 5 7 ^ { a } )}$ & $\mathbf{4 6 7}$ & $\mathbf{4 5}(\mathbf{9 , 6 3 )}$ & $\mathbf{6 2 ( 6 . 7 ^ { b } )}$ \\
\hline MS & 16 & $15(93,75)^{a}$ & 79 & $14(17,72)$ & $15\left(24,19^{c}\right)$ \\
MT & 10 & $10(100)^{a}$ & 141 & $6(4,25)$ & $10\left(16,12^{c}\right)$ \\
GO & 33 & $28(84,84)^{a}$ & 246 & $24(9,75)$ & $28\left(45,16^{c}\right)$ \\
DF & 11 & $9(81,81)^{a}$ & 1 & $1(100)$ & $9\left(14,51^{c}\right)$ \\
\hline BRASIL & $\mathbf{9 8 8}$ & $\mathbf{9 3 2}\left(\mathbf{9 4 , 3 3 ^ { a } )}\right.$ & $\mathbf{5 . 5 7 0}$ & $\mathbf{7 7 9}(\mathbf{1 3 , 9 8 )}$ & $\mathbf{9 3 2}(\mathbf{1 0 0})$ \\
\hline
\end{tabular}

(a) Frequência relativa de CEO participantes do PMAQ, em relaçâo ao total de CEO implantados no Brasil.

(b) Frequência relativa ao total de CEO participantes do PMAQ por regiāo, em relação ao total de CEO participantes do PMAQ no Brasil.

(c) Frequência relativa ao total de CEO participantes do PMAQ por estado, em relação ao total de CEO participantes do PMAQ no Brasil.

Fonte: Dados do IBGE, 2013; PMAQ-CEO, 2014; SAGE, 2019.

A distribuição territorial dos CEO demonstrou desigualdades inter e intrarregional quanto à implantação e à cobertura desses serviços. No território nacional, somente 13,98\% dos municípios brasileiros são cobertos por CEO, a maioria Tipo II (50,9\%). A regiáo Norte possui o menor número de serviços e a região Nordeste o maior. Entretanto, estas duas macrorregióes apresentaram a maior deficiência, a nível nacional, quanto à estrutura, equipamentos e insumos que influenciam diretamente no serviço ofertado. As regiōes Sul e Centro-Oeste apresentaram cobertura inferior a $10 \%$.

O Distrito Federal e os estados do Rio de Janeiro e Sáo Paulo apresentaram a melhor cobertura de serviços implantados, com 100\%, 45,65\% e 22,32\% dos municípios cobertos, respectivamente. Os estados do Acre, Rio Grande do Sul e Mato Grosso apresentaram cobertura inferior a 5\%. 


\section{Módulo 1 da Avaliação Externa}

No primeiro módulo, que avaliou itens relacionados à infraestrutura e recursos materiais, foi realizada a seleção aleatória de 18 itens referentes aos Equipamentos, 16 itens referentes aos Insumos e 16 itens referentes aos Instrumentais, com o objetivo de identificar se os $\mathrm{CEO}$ estavam adequados. $\mathrm{O}$ resultado desta análise evidenciou que menos de $1,1 \%$ dos $\mathrm{CEO}$ brasileiros apresentavam todos os equipamentos avaliados, menos de $40 \%$ dos serviços estavam adequados quanto aos itens insumos e instrumentais, somente o item relacionado aos materiais impressos foi satisfatório, acima de $95 \%$.

Entretanto, como a análise nacional selecionou itens aleatórios para avaliar a adequação dos CEO quanto aos recursos materiais de maneira geral, este estudo, para contemplar uma análise de adequação dos serviços com foco à Estomatologia, avaliou a adequação dos CEO quanto aos recursos materiais em uso ou no estoque utilizados nos procedimentos realizados nessa especialidade, o que pode ser observado na Tabela 2.

Tabela 2. Frequência absoluta e relativa de CEO com disponibilidade recursos materiais, utilizados no cumprimento das atividades na especialidade de Estomatologia, em uso ou no estoque, por Região e Unidades Federativas (UF).

\begin{tabular}{|c|c|c|c|c|c|c|c|}
\hline $\begin{array}{c}\text { Regiōes e } \\
\text { Estados }\end{array}$ & $\begin{array}{c}\text { CEO } \\
\text { participantes } \\
\text { do PMAQ }\end{array}$ & 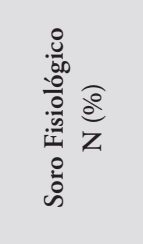 & 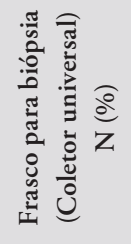 & 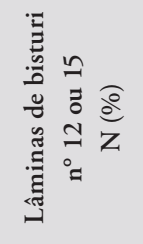 & 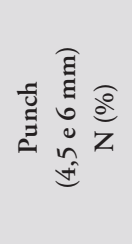 & 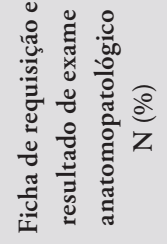 & 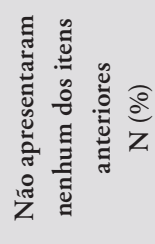 \\
\hline $\begin{array}{c}\text { Região } \\
\text { Norte }\end{array}$ & 60 & $19(31,66)$ & $6(10)$ & $19(31,66)$ & $4(6,66)$ & $30(50)$ & $13(21,66)$ \\
\hline $\mathrm{RO}$ & 7 & $1(14,28)$ & $0(0)$ & $0(0)$ & $0(0)$ & $2(28,57)$ & $4(57,14)$ \\
\hline $\mathrm{AC}$ & 2 & $0(0)$ & $0(0)$ & $1(50)$ & $0(0)$ & $2(100)$ & $0(0)$ \\
\hline $\mathrm{AM}$ & 11 & $2(18,18)$ & $0(0)$ & $4(36,36)$ & $2(18,18)$ & $4(36,36)$ & $4(36,36)$ \\
\hline $\mathrm{RR}$ & 1 & $1(100)$ & $0(0)$ & $1(100)$ & $0(0)$ & $0(0)$ & $0(0)$ \\
\hline PA & 29 & $11(37,93)$ & $5(17,24)$ & $7(24,13)$ & $2(6,89)$ & $17(58,62)$ & $3(10,34)$ \\
\hline $\mathrm{AP}$ & 3 & $2(66,66)$ & $0(0)$ & $2(66,66)$ & $0(0)$ & $2(66,66)$ & $0(0)$ \\
\hline TO & 7 & $2(28,57)$ & $1(14,28)$ & $4(57,14)$ & $0(0)$ & $3(42,85)$ & $2(28,57)$ \\
\hline
\end{tabular}




\begin{tabular}{|c|c|c|c|c|c|c|c|}
\hline $\begin{array}{l}\text { Regióes e } \\
\text { Estados }\end{array}$ & $\begin{array}{c}\text { CEO } \\
\text { participantes } \\
\text { do PMAQ }\end{array}$ & 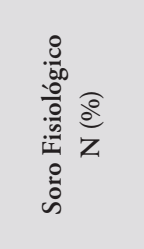 & 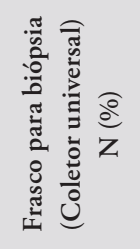 & 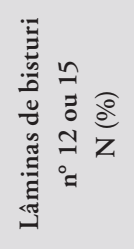 & 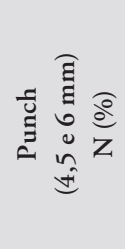 & 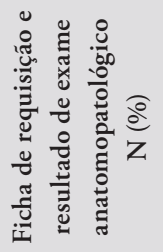 & 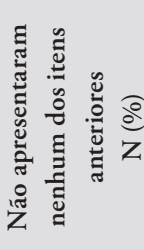 \\
\hline $\begin{array}{c}\text { Regiáo } \\
\text { Nordeste }\end{array}$ & 357 & $84(23,52)$ & $56(15,68)$ & $\begin{array}{c}140 \\
(39,21)\end{array}$ & $15(4,20)$ & $183(51,26)$ & $69(19,32)$ \\
\hline MA & 26 & $3(11,53)$ & $3(11,53)$ & $12(46,15)$ & $0(0)$ & $9(34,61)$ & $7(26,92)$ \\
\hline PI & 28 & $5(17,85)$ & $5(17,85)$ & $7(25)$ & $4(14,28)$ & $13(46,42)$ & $4(14,28)$ \\
\hline CE & 80 & $17(21,25)$ & $8(10)$ & $37(46,25)$ & $1(1,25)$ & $46(57,5)$ & $14(17,5)$ \\
\hline RN & 23 & $7(30,43)$ & $4(17,39)$ & $7(30,43)$ & $3(13,04)$ & $16(69,56)$ & $2(8,69)$ \\
\hline $\mathrm{PB}$ & 53 & $18(33,96)$ & $10(18,86)$ & $25(47,16)$ & $1(1,88)$ & $33(62,26)$ & $8(15,09)$ \\
\hline PE & 40 & $8(20)$ & $10(25)$ & $12(30)$ & $3(7,5)$ & $19(47,5)$ & $10(25)$ \\
\hline $\mathrm{AL}$ & 22 & $4(18,18)$ & $3(13,63)$ & $11(50)$ & $0(0)$ & $7(31,81)$ & $4(18,18)$ \\
\hline SE & 10 & $2(20)$ & $2(20)$ & $2(20)$ & $0(0)$ & $9(90)$ & $0(0)$ \\
\hline BA & 75 & $20(26,66)$ & $11(14,66)$ & $27(36)$ & $3(11,11)$ & $31(41,33)$ & $20(26,66)$ \\
\hline $\begin{array}{l}\text { Regiáo } \\
\text { Sudeste }\end{array}$ & 337 & $79(23,44)$ & $51(15,13)$ & $\begin{array}{c}117 \\
(34,71)\end{array}$ & $24(7,12)$ & $282(83,67)$ & $23(6,82)$ \\
\hline MG & 83 & $22(26,50)$ & $16(18,60)$ & $29(34,93)$ & $4(4,81)$ & $70(84,33)$ & $6(7,22)$ \\
\hline ES & 9 & $2(22,22)$ & $1(11,11)$ & $1(11,11)$ & $0(0)$ & $8(88,88)$ & $1(11,11)$ \\
\hline RJ & 66 & $18(27,27)$ & $11(16,66)$ & $24(36,36)$ & $10(15,15)$ & $45(68,18)$ & $9(13,63)$ \\
\hline SP & 179 & $37(20,67)$ & $23(12,84)$ & $63(35,19)$ & $10(5,58)$ & $159(88,82)$ & $7(3,91)$ \\
\hline $\begin{array}{c}\text { Região } \\
\text { Sul }\end{array}$ & 116 & $30(25,86)$ & $23(19,82)$ & $45(38,79)$ & $11(9,48)$ & $99(85,34)$ & $5(4,31)$ \\
\hline PR & 48 & $14(29,16)$ & $11(22,91)$ & $18(37,5)$ & $5(10,41)$ & $41(85,41)$ & $3(6,25)$ \\
\hline SC & 43 & $9(20,93)$ & $8(18,60)$ & $15(34,88)$ & $4(9,30)$ & $41(95,34)$ & $0(0)$ \\
\hline RS & 25 & 7 (28) & $4(16)$ & $12(48)$ & $2(8)$ & $17(68)$ & $2(8)$ \\
\hline
\end{tabular}

continua... 


\begin{tabular}{|c|c|c|c|c|c|c|c|}
\hline $\begin{array}{l}\text { Regióes e } \\
\text { Estados }\end{array}$ & $\begin{array}{c}\text { CEO } \\
\text { participantes } \\
\text { do PMAQ }\end{array}$ & 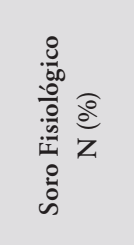 & 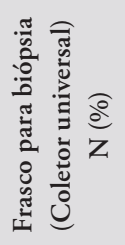 & 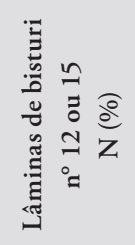 & 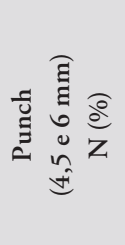 & 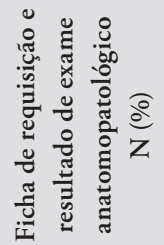 & 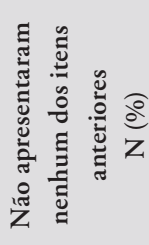 \\
\hline $\begin{array}{c}\text { Região } \\
\text { Centro- } \\
\text { Oeste }\end{array}$ & 62 & $16(25,80)$ & $9(14,51)$ & $18(29,03)$ & $5(8,06)$ & $56(90,32)$ & $5(8,06)$ \\
\hline MS & 15 & $5(33,33)$ & 0 & $5(33,33)$ & $1(6,66)$ & $13(86,66 \mid 0$ & $3(20)$ \\
\hline MT & 10 & $1(10)$ & $1(10)$ & $3(30)$ & $0(0)$ & $10(100)$ & $0(0)$ \\
\hline $\mathrm{GO}$ & 28 & $8(28,57)$ & $5(17,85)$ & $9(32,14)$ & $2(7,14)$ & $24(85,71)$ & $2(7,14)$ \\
\hline DF & 9 & $2(22,22)$ & $3(33,33)$ & $1(11,11)$ & $2(22,22)$ & $9(100)$ & $0(0)$ \\
\hline BRASIL & 932 & $\begin{array}{c}228 \\
(24,46)\end{array}$ & $\begin{array}{c}145 \\
(15,55)\end{array}$ & $\begin{array}{c}339 \\
(36,37)\end{array}$ & $59(6,33)$ & $650(69,74)$ & $115(12,33)$ \\
\hline
\end{tabular}

$N$ Frequência absoluta de CEO com disponibilidade recursos materiais utilizados no cumprimento das atividades na especialidade de Estomatologia.

(\%) Frequência relativa de CEO com disponibilidade recursos materiais utilizados no cumprimento das atividades na especialidade de Estomatologia.

Fonte: Microdados coletados do PMAQ - CEO (Brasil, 2014).

Identificou-se que 704 (75,53\%) CEO brasileiros não tinham soro fisiológico para a realização do procedimento e transporte da peça de biópsia; 787 (84,44\%) CEO não possuíam frasco de biópsia; $593(63,62 \%)$ não possuíam lâmina de bisturi número 12 ou 15; 873 (93,66\%) CEO não tinham punch; 282 (30,25\%) CEO não tinham ficha de requisição e resultado de exame anatomopatológico em uso ou no estoque e 115 (12,33\%) CEO não apresentavam nenhum destes recursos materiais.

\section{Módulo 2 da Avaliação Externa}

No segundo módulo, que avaliou itens relacionados ao serviço e aos profissionais, foi observado que o acesso do brasileiro à assistência especializada era predominantemente realizado de acordo com a hierarquização da rede de serviços do SUS, sendo via ficha de encaminhamento/referência, entre UBS e CEO, e a espera pelo tratamento variava de uma semana a um mês. Para a especialidade de Estomatologia, analisada neste estudo, a situação foi semelhante, pois 574 (61,58\%) CEO recebiam usuários referenciados da UBS, majoritariamente sem cotas para a 
especialidade, com 808 (86,69\%) CEO sem cotas pré-definidas; e o tempo de espera médio nacional era de aproximadamente 7 a 8 dias.

Apesar do fluxo entre a atenção básica e especializada ser um aspecto positivo, este estudo identificou que menos da metade dos serviços da região Norte e Nordeste possuíam protocolo clínico de encaminhamento para a especialidade de Estomatologia, em contraste com as demais regiôes, demonstrado na Tabela 3. Além disso, os dados demonstraram que a capacitação profissional para a prevenção do câncer de boca, um dos itens do matriciamento $\mathrm{AB}$ - CEO, não apresentava frequência superior a $50 \%$ dos serviços.

Tabela 3. Frequência absoluta (N) e relativa (\%) referentes a existência de protocolos clínicos de encaminhamento para Estomatologia e ao cumprimento das atividades da especialidade por Regiáo e Unidades Federativas (UF). Brasil, 2014.

\begin{tabular}{ccccc}
\hline Regióes e Estados & $\begin{array}{c}\text { CEO com } \\
\text { protocolo de } \\
\text { encaminhamento } \\
\text { para Estomatologia }\end{array}$ & Estomatologia (diagnóstico do câncer de boca) \\
\hline N (\%) & Faz biópsia & $\begin{array}{c}\text { Tem referência p/ } \\
\text { anatomopatológico }\end{array}$ & $\begin{array}{c}\text { Tem registro } \\
\text { dos casos }\end{array}$ \\
\hline Regiáo Norte & $\mathbf{1 2 ( 2 0 , 3 )}$ & $\mathbf{3 9}(\mathbf{6 6 , 1 )}$ & $\mathbf{3 8}(\mathbf{6 4 , 4})$ & $\mathbf{1 1}(\mathbf{1 8 , 6})$ \\
\hline RO & $0(0,0)$ & $3(42,9)$ & $3(42,9)$ & $3(42,9)$ \\
AC & $2(100)$ & $2(100,0)$ & $2(100,0)$ & $0(0,0)$ \\
AM & $1(9,1)$ & $6(54,5)$ & $6(54,5)$ & $1(9,1)$ \\
RR & $0(0,0)$ & $0(0,0)$ & $0(0,0)$ & $0(0,0)$ \\
PA & $4(14,3)$ & $20(71,4)$ & $20(71,4)$ & $5(17,9)$ \\
AP & $0(0)$ & $3(100,0)$ & $2(66,7)$ & $1(33,3)$ \\
TO & $5(7,4)$ & $5(71,4)$ & $5(71,4)$ & $1(14,3)$ \\
\hline Regiáo Nordeste & $\mathbf{1 2 0 ( 3 3 , 8 )}$ & $\mathbf{2 6 9 ( 7 5 , 8 )}$ & $\mathbf{2 2 2}(62,5)$ & $\mathbf{1 2 6}(35,5)$ \\
\hline MA & $5(19,2)$ & $16(61,5)$ & $10(38,5)$ & $3(11,5)$ \\
PI & $17(60,7)$ & $18(64,3)$ & $16(57,1)$ & $7(25,0)$ \\
CE & $30(38,0)$ & $59(74,7)$ & $47(59,5)$ & $34(43,0)$ \\
\hline
\end{tabular}

continua... 


\begin{tabular}{|c|c|c|c|c|}
\hline \multirow[t]{3}{*}{ Regióes e Estados } & \multirow{3}{*}{$\begin{array}{c}\text { CEO com } \\
\text { protocolo de } \\
\text { encaminhamento } \\
\text { para Estomatologia }\end{array}$} & \multicolumn{3}{|c|}{ Estomatologia (diagnóstico do câncer de boca) } \\
\hline & & Faz biópsia & $\begin{array}{l}\text { Tem referência p/ } \\
\text { anatomopatológico }\end{array}$ & $\begin{array}{c}\text { Tem registro } \\
\text { dos casos }\end{array}$ \\
\hline & & N (\%) & N (\%) & N (\%) \\
\hline $\mathrm{RN}$ & $8(34,8)$ & $22(95,6)$ & $20(87,0)$ & $11(47,8)$ \\
\hline $\mathrm{PB}$ & $14(26,4)$ & $39(73,6)$ & $31(58,5)$ & $13(24,5)$ \\
\hline PE & $18(45,0)$ & $30(75,0)$ & $28(70,0)$ & $12(30,0)$ \\
\hline $\mathrm{AL}$ & $5(22,7)$ & $19(86,4)$ & $14(63,6)$ & $8(36,4)$ \\
\hline SE & $1(10,0)$ & $10(100,0)$ & $7(70,0)$ & $5(50,0)$ \\
\hline BA & $22(29,7)$ & $56(75,7)$ & $49(66,2)$ & $33(44,6)$ \\
\hline Regiáo Sudeste & $231(68,5)$ & $294(87,2)$ & $290(86,0)$ & $210(62,3)$ \\
\hline MG & $55(66,3)$ & $72(86,7)$ & $72(86,7)$ & $54(65,1)$ \\
\hline ES & $4(44,4)$ & $8(88,9)$ & $8(88,9)$ & $3(33,3)$ \\
\hline RJ & $41(62,1)$ & $57(86,4)$ & $54(81,8)$ & $32(48,5)$ \\
\hline SP & $131(73,2)$ & $157(87,7)$ & $156(87,1)$ & $121(67,6)$ \\
\hline Regiáo Sul & $71(60,7)$ & $104(88,9)$ & $102(87,2)$ & $59(50,4)$ \\
\hline PR & $34(69,4)$ & $45(91,8)$ & $44(89,8)$ & $28(57,1)$ \\
\hline SC & $26(60,5)$ & $40(93,0)$ & $39(90,7)$ & $21(48,8)$ \\
\hline $\mathrm{RS}$ & $11(44,0)$ & $19(76,0)$ & $19(76,0)$ & $10(40,0)$ \\
\hline $\begin{array}{c}\text { Regiáo Centro- } \\
\text { Oeste }\end{array}$ & $36(58,1)$ & $59(95,2)$ & $55(88,7)$ & $30(48,4)$ \\
\hline MS & $13(86,7)$ & $15(100,0)$ & $13(86,7)$ & $9(60,0)$ \\
\hline MT & $1(10,0)$ & $10(100,0)$ & $10(100,0)$ & $4(40,0)$ \\
\hline GO & $18(64,3)$ & $26(92,9)$ & $24(85,7)$ & $14(50,0)$ \\
\hline DF & $4(44,4)$ & $8(88,9)$ & $8(88,9)$ & $3(33,3)$ \\
\hline BRASIL & $470(50,5)$ & $765(82,3)$ & $707(76,0)$ & $436(46,9)$ \\
\hline
\end{tabular}

Fonte: Microdados coletados do PMAQ - CEO (Brasil, 2014). 
Em relação aos profissionais, também avaliados no segundo módulo, foi observado que, dos 932 CEO participantes do PMAQ-CEO, somente 65,55\% (611), apresentavam Cirurgióes Dentistas atuantes na especialidade de Estomatologia. Embora haja a obrigatoriedade de profissionais atuantes na especialidade, na região Norte somente $33,33 \%$ (20) dos CEO apresentavam dentistas no serviço de Estomatologia, estando os estados do Acre, Amazonas e Roraima sem profissionais na especialidade, observado na Tabela 4.

Tabela 4. Relação de Centros de Especialidades Odontológicas (CEO) com serviço de Estomatologia, frequência absoluta $(\mathrm{N})$ e relativa (\%) de Cirurgióes Dentistas atuantes na especialidade e média da carga horaria semanal por Regiấo e Unidades Federativas (UF).

\begin{tabular}{|c|c|c|c|c|}
\hline Regióes e Estados & $\begin{array}{l}\text { Quantidade de } \\
\text { CD atuantes em } \\
\text { Estomatologia }\end{array}$ & $\begin{array}{l}\text { Carga Horária } \\
\text { semanal média } \\
\text { dos CD atuantes } \\
\text { em Estomatologia }\end{array}$ & $\begin{array}{c}\text { CEO que tem } \\
\text { CD atuantes em } \\
\text { Estomatologia } \\
\text { N (\%) }\end{array}$ & $\begin{array}{c}\text { Total de CEO } \\
\text { participantes do } \\
\text { PMAQ }\end{array}$ \\
\hline Regiáo Norte & 26 & 25,47 & $20(33,33)$ & 60 \\
\hline $\mathrm{RO}$ & 2 & 30 & $2(28,57)$ & 7 \\
\hline $\mathrm{AC}$ & 0 & 0 & $0(0)$ & 2 \\
\hline AM & 0 & 0 & $0(0)$ & 11 \\
\hline $\mathrm{RR}$ & 0 & 0 & $0(0)$ & 1 \\
\hline PA & 19 & 24,15 & $13(44,82)$ & 29 \\
\hline $\mathrm{AP}$ & 2 & 30 & $2(66,66)$ & 3 \\
\hline $\mathrm{TO}$ & 3 & 26,66 & $3(42,85)$ & 7 \\
\hline Regiáo Nordeste & 236 & 19,88 & $196(54,90)$ & 357 \\
\hline MA & 11 & 15,44 & $9(34,61)$ & 26 \\
\hline PI & 24 & 3,69 & $13(46,42)$ & 28 \\
\hline CE & 68 & 22,19 & $56(70)$ & 80 \\
\hline $\mathrm{RN}$ & 4 & 17,5 & $4(17)$ & 23 \\
\hline $\mathrm{PB}$ & 33 & 17,73 & $30(56,60)$ & 53 \\
\hline PE & 33 & 22,96 & $29(72,5)$ & 40 \\
\hline $\mathrm{AL}$ & 4 & 26,66 & $3(13,63)$ & 22 \\
\hline SE & 1 & 30 & $1(10)$ & 10 \\
\hline $\mathrm{BA}$ & 58 & 22,05 & $51(68)$ & 75 \\
\hline
\end{tabular}




\begin{tabular}{|c|c|c|c|c|}
\hline Regióes e Estados & $\begin{array}{l}\text { Quantidade de } \\
\text { CD atuantes em } \\
\text { Estomatologia }\end{array}$ & $\begin{array}{l}\text { Carga Horária } \\
\text { semanal média } \\
\text { dos CD atuantes } \\
\text { em Estomatologia }\end{array}$ & $\begin{array}{c}\text { CEO que tem } \\
\text { CD atuantes em } \\
\text { Estomatologia } \\
\mathrm{N}(\%)\end{array}$ & $\begin{array}{c}\text { Total de CEO } \\
\text { participantes do } \\
\text { PMAQ }\end{array}$ \\
\hline Região Sudeste & 313 & 20,99 & $263(78,04)$ & 337 \\
\hline MG & 74 & 22,36 & $60(72,28)$ & 83 \\
\hline ES & 4 & 26,66 & $3(33,33)$ & 9 \\
\hline RJ & 65 & 19,71 & $56(84,84)$ & 66 \\
\hline SP & 170 & 20,59 & $144(80,44)$ & 179 \\
\hline Regiáo Sul & 170 & 14,12 & $86(74,13)$ & 116 \\
\hline PR & 51 & 15,91 & $38(79,16)$ & 48 \\
\hline SC & 52 & 14,65 & $35(81,39)$ & 43 \\
\hline RS & 67 & 14,48 & $13(52)$ & 25 \\
\hline $\begin{array}{c}\text { Região Centro- } \\
\text { Oeste }\end{array}$ & 83 & 25,89 & $46(74,19)$ & 62 \\
\hline MS & 23 & 23,75 & $12(80)$ & 15 \\
\hline MT & 4 & 22,5 & $4(40)$ & 10 \\
\hline GO & 43 & 25,86 & $22(78,57)$ & 28 \\
\hline DF & 13 & 30,87 & $8(88,88)$ & 9 \\
\hline BRASIL & 828 & 20,88 & $611(65,55)$ & 932 \\
\hline
\end{tabular}

Fonte: Microdados coletados do PMAQ - CEO (Brasil, 2014).

Foram observadas variaçóes entre as regióes quanto à formação e à carga horária de atuação dos dentistas especialistas em Estomatologia. Nas regióes Norte e Sul, a maioria desses profissionais possuíam somente a graduação (com média de 32,2 e 0,6 respectivamente) diferindo da média nacional $(0,2)$ e demais regióes, onde a maioria dos profissionais possuíam atualização e/ou são especializados. Nas regiôes Nordeste e Sul, a média de carga horária de atuação semanal dos profissionais era inferior a 20 horas, resultando em menos 4 horas diárias por semana de atuação na especialidade, contrastando com as demais regióes.

Este estudo identificou que o cumprimento das principais atividades na especialidade de Estomatologia, na maioria dos serviços do país, ocorria de forma satisfatória (Tabela 3). Em 765 (82,3\%) dos CEO do Brasil a biópsia era realizada, 
sendo que em $37,90 \%$ dos CEO acontecia no mesmo momento da consulta, e em $442(57,77 \%)$ no período de 1 dia até 15 dias após a consulta.

A referência laboratorial para o exame histopatológico também pode ser considerada satisfatória, pois ocorria em mais de $70 \%$ dos serviços brasileiros. Apesar disso, identificou-se que em $28(3,66 \%)$ CEO a realização da biópsia variava de 15 a 60 dias, que em $482(63,01 \%)$ CEO o recebimento do laudo do patologista demorava mais de 15 dias e que o estado de Roraima não realizava nenhuma atividade da especialidade.

\section{Discussão}

A análise do cenário da saúde bucal, de modo geral, evidencia melhora na cobertura populacional após a implementação da PNSB, com a ampliação da atenção básica e implantação dos Centros de Especialidades Odontológicas. Dados da Pesquisa Nacional por Amostra de Domicílio (PNAD), demonstraram que, em 2003, 15,9\% (27,9 milhôes) da população brasileira nunca havia ido ao dentista, enquanto que, em 2008, após a implantação da PNSB, este valor foi reduzido para 11,7\% (22,1 milhôes) (IBGE, 2005; IBGE 2008).

Antes da implantação da PNSB, a oferta da assistência especializada era muito reduzida e, muitas vezes, a resolubilidade de problemas bucais complexos não acontecia, ou, quando solucionados, resultavam em medidas irreversíveis na Atenção Básica, como, por exemplo, a extração do elemento dentário (PAIM, 2011). Neste sentido, o Brasil Sorridente, foi a primeira política de saúde que o governo federal passou a oferecer amplamente à população, tratamento odontológico especializado no SUS (PUCA JÚNIOR, 2006; CHAVES et al., 2011).

Entretanto, mesmo com os avanços da PNSB relacionados à atençấo básica e especializada, a assistência odontológica não é equânime em todo o Brasil, o que reflete diretamente no padrão epidemiológico desigual das doenças bucais mais prevalentes, nas diferentes macrorregióes brasileiras (DUARTE et al., 2002). Como exemplo desta realidade, os dados epidemiológicos dos últimos levantamentos nacionais em saúde bucal, realizados no Brasil no período de 2003 a 2010, demonstraram melhora da condição de saúde bucal dos brasileiros, com o declínio da cárie dentária a nível nacional, em contraste com o aumento da doença na região Norte do país (BRASIL, 2004b; BRASIL, 2010; CHAVES et al., 2017). 
Embora as desigualdades da assistência odontológica já pudessem ser observadas nos dados epidemiológicos, ou ainda em dados relacionados à implantação dos serviços e de cobertura populacional, disponíveis pelo MS e IBGE, somente com a implementação do PMAQ-CEO houve, de fato, o estabelecimento de metodologias consistentes capazes de identificar disparidades, fragilidades, iniquidades e potencialidades, que servissem de diagnóstico e subsídio para a gestão e melhoria dos serviços (CASOTTO et al., 2014; FACCHINI et al., 2018).

Em relação à especialidade de Estomatologia, analisada neste estudo, foi observado que, apesar desta especialidade ser mínima e obrigatória em todos os serviços de atenção secundária no país, apresentou as maiores deficiências entre as demais especialidades obrigatórias nos CEO, observadas nos 2 módulos da fase de Avaliação Externa. Os itens avaliados referentes ao terceiro módulo da avaliação externa não foram abordados, pois contemplaram o serviço em sua totalidade, e não circunscrito à especialidade de Estomatologia, objeto desse estudo.

No módulo 1, foi observado que os serviços brasileiros se encontravam insuficientemente equipados e a regiâo Nordeste era a mais crítica quanto ao suprimento de recursos materiais relacionados a todas as especialidades. Quanto aos itens relacionados à Estomatologia, analisados nesse estudo, identificou-se que a falta de recursos ainda é mais acentuada, pois em mais de $60 \%$ dos CEO não havia materiais, em uso ou no estoque, fundamentais para a realização de biópsia, que é o procedimento mais comum na especialidade. Nesse contexto, a região Norte foi a mais afetada quanto aos recursos materiais utilizados especificamente na especialidade de Estomatologia.

Isso pode justificar a considerável frequência (30\%) de profissionais dos CEO do País que relataram deixar de atender por falta de insumos/instrumental e equipamentos, dentre os quais encontraram-se os atuantes na Estomatologia. As deficiências observadas no suprimento de recursos materiais comprometem a assistência especializada e a falta de assistência na especialidade de Estomatologia pode resultar em morbidades irreversíveis, afetando diretamente a qualidade de vida dos pacientes, ou ainda vitimá-los a óbito.

No módulo 2, este estudo identificou falhas graves de fluxo na rede de serviços do SUS; pois, apesar do acesso aos CEO observado ser majoritariamente via Encaminhamento/Referência da $A B$, a existência de protocolo clínico pactuado entre $\mathrm{AB}-\mathrm{CEO}$ para especialidade de Estomatologia, era a menor entre as especialidades 
básicas e presente em menos da metade dos serviços da regiáo Norte e Nordeste, contrastando com as demais macrorregióes. Além disso, o apoio matricial, que corresponde ao suporte e compartilhamento de informaçóes para o diagnóstico de câncer entre AB-CEO não alcançava 50\% dos serviços, em todo o País, retardando o diagnóstico e tratamento da doença.

Também foi observado que, apesar da maioria dos profissionais atuantes em Estomatologia serem especialistas ou com aperfeiçoamento (75,54\%), a especialidade possui a menor quantidade de profissionais atuantes, menor carga horária e o cumprimento das atividades inerentes à especialidade, não era realizado em todos os CEO do País. Além disso, na região Norte, menos de 35\% dos CEO apresentavam esses profissionais, sendo os estados do Acre, Amazonas e Roraima descobertos, mesmo que a taxa média de crescimento de câncer bucal sejam de 3,54 e 4,93, para ambos os gêneros, no estado de Acre e Roraima, respectivamente e a estimativa de 90 novos casos de câncer bucal para homens e 30 para mulheres em 2020, no Amazonas, com taxa média de crescimento de câncer bucal de 4,42, para ambos os gêneros, no triênio 2020-2022 (INCA, 2019).

Quanto ao cumprimento das atividades de Estomatologia, apesar da frequência de serviços que realizavam o exame de biópsia em tempo satisfatório, bem como a adequação quanto à referência laboratorial para o exame histopatológico constituírem aspectos positivos na avaliação da especialidade, em mais de $60 \%$ dos $\mathrm{CEO}$ a entrega do laudo ultrapassava 15 dias, podendo chegar a 2 meses de espera em alguns locais. Esta demora do laudo pode atrasar o tratamento e contribuir para o agravo do câncer de boca, que foi registrado em 46,9\% dos CEO, evidenciando a frequência da enfermidade nos serviços.

Outro dado preocupante evidenciado neste estudo refere-se ao registro de casos confirmados para câncer bucal. Os dados epidemiológicos disponíveis na literatura científica sobre a doença são majoritariamente oriundos da atenção secundária, avaliados no PMAQ-CEO, e Centros de Referências de Atenção Terciária, como Unidades de Assistência de Alta Complexidade em Oncologia (UNACON) e os Centros de Assistência de Alta Complexidade em Oncologia (CACON), integrantes de complexos hospitalares de referências e publicaçôes do INCA.

Entretanto, este estudo identificou falhas graves no fluxo de assistência, evidenciadas pela avaliação externa do PMAQ-CEO. Inúmeros usuários, muitas 
vezes, não lograram ser assistidos em todos os níveis de atenção, ou seja, não passavam da atenção básica para a atenção secundária e terciária, onde a maioria dos casos são registrados (FACCHINI et al., 2018).

Como exemplificação quanto à deficiência na cobertura, estudos mostram que usuários da região Norte morando em municípios populosos que deveriam ser cobertos por CEO relataram não buscar assistência em virtude das barreiras na acessibilidade geográfica a essas unidades de saúde (CASOTTO et al., 2014). Como deficiência dos serviços, nos municípios que têm cobertura de CEO foi observado que mesmo nos serviços onde o acesso ocorre mediante referenciamento, os usuários afirmaram que possuir ficha de referência não garantia a realização de procedimentos, pois muitas vezes os CEO não têm profissionais ou recursos para a realização do procedimento especializado (GOES et al., 2012; MACHADO et al., 2015).

Embora o diagnóstico da atenção secundária seja fundamental para a melhoria dos serviços, na literatura científica disponível, foi observado uma escassez de estudos acerca da avaliação em saúde bucal na atenção especializada, em sua totalidade. No que se refere objetivamente às especialidades odontológicas ofertadas nos CEO, este estudo identificou a inexistência de literatura científica que avaliasse a especialidade de Estomatologia em serviços especializados.

De acordo com os dados avaliados nesse estudo, ressalta-se que a assistência odontológica secundária no Brasil ainda é marcada pela falta de cobertura, ou por ineficiência dos serviços onde há CEO, comprometendo o acesso ao tratamento pelo usuário. Para a especialidade de Estomatologia essa realidade é ainda mais grave em comparação às outras especialidades obrigatórias, tanto pelas iniquidades observadas serem mais significativas, quanto pela importância da especialidade no diagnóstico do câncer de boca.

Essa realidade somente pôde ser observada pelos dados obtidos no PMAQ$\mathrm{CEO}$, demonstrando assim a importância da adesão e participação dos serviços em programas de avaliação do acesso, desempenho e qualidade para a melhora dos serviços prestados à população. Antes do PMAQ-CEO, os dados disponíveis somente evidenciavam as disparidades e deficiências quanto à cobertura de CEO no país e, após a implementação do programa, houve a possibilidade do conhecimento e diagnóstico dos serviços prestados por estes CEO implantados. 
Para a especialidade de estomatologia, foco desse estudo, identificou-se que além da cobertura deficiente nas macrorregiōes brasileiras, as principais deficiências observadas eram quanto ao cumprimento de atividades da especialidade, a falta de profissionais e de recursos materiais.

Dos 932 serviços avaliados, cerca de 82\% (765 CEO) realizavam a biópsia, que é o principal exame de diagnóstico para o câncer bucal e obrigatório nesses serviços, somente 611cirurgiôes-dentistas atuavam para a realização, interpretação e confirmação diagnóstica dessa afecção, o que não corresponderia a 1 profissional para cada CEO do país. Além disso, cerca de 30\% desses profissionais deixaram de atender por falta de recursos materiais, pois somente $342 \mathrm{CEO}$ estavam adequados quanto aos materiais e insumos para o atendimento e assistência à população.

Assim, tendo em vista que o câncer bucal se apresenta como uma lesão invasiva que geralmente resulta em morbidades locais, como cirurgias radicais ou amputaçôes de maxila e mandíbula e morbidades sistêmicas resultantes do avanço da doença, a falta de acesso ao tratamento especializado, evidenciado nesse estudo, pode justificar a elevada estimativa de óbito relacionada ao câncer de boca no Brasil.

Destaca-se como limitação deste estudo a utilização de dados secundários, os quais algumas vezes não apresentaram uniformidade de identificação dos estabelecimentos visitados entre os módulos, permitindo a análise e interpretação dos dados de cada módulo apenas individualmente. Outra fragilidade importante é o fato de terem tido $\mathrm{CEO}$ não avaliados no país durante o $1^{\circ}$ ciclo do $\mathrm{PMAQ-CEO}$, visto isso não trazer o retrato da especialidade na totalidade dos serviços secundários no país. Contudo, esta lacuna da avaliação pode ser contemplada nos próximos ciclos do programa.

Evidencia-se como potencialidades a constatação, por meio dos dados analisados, quanto às deficiências da especialidade de estomatologia no país, tendo em vista a inexistência de estudos anteriores que diagnosticassem a oferta da especialidade no âmbito do SUS. Dessa forma, ressalta-se a importância do PMAQ-CEO como método para institucionalizar a avaliação em saúde, induzindo mudanças necessárias para melhoria do acesso e assistência à saúde.

\section{Conclusão}

A participação dos CEO no PMAQ possibilitou o diagnóstico da atenção especializada quanto ao acesso e qualidade. A atenção secundária no Brasil, 
institucionalizadas nos CEO pela PNSB, ainda assiste à uma pequena parcela populacional e apresenta diferenças na implantação, recursos materiais e humano, desses serviços no Brasil. Apesar do aumento na afecção e estimativa do câncer de boca, a especialidade de Estomatologia apresentou considerável deficiência, caracterizando esta especialidade como uma das mais afetadas entre as especialidades básicas e obrigatórias na atenção especializada, o que pode contribuir para o aumento de agravos e morbidades bucais. Assim, os pontos fortes e fragilidades coletados nesta primeira avaliação mencionados aqui podem ser decisivos para contribuir para os futuros ciclos de avaliação do PMAQ-CEO, com o objetivo de apontar a situação da Estomatologia, na atenção secundária, transformar deficiências em potencialidades, a fim de garantir o acesso dos usuários e a qualidade dos serviços ofertados à população.

\section{Referências}

BALDANI, M.H.; RIBEIO, A.E.; GONÇALVES, J.R.S.N.; DIETTERICH, R.G. Processo de trabalho em saúde bucal na atenção básica: desigualdades intermunicipais evidenciadas pelo PMAQ-AB. Rev Saúde e Debate. v. 42, n. spel, p. 145-162, 2018.

BRASIL. Ministério da Saúde. Portaria No 1.444 de 28 de dezembro de 2000. Estabelece incentivo financeiro para a reorganização da Atenção em Saúde Bucal prestada nos municípios por meio do Programa Saúde da Família. Diário Oficial da União, Brasília, 29 dez. 2000. Disponível em: https:/www.camara.leg.br/proposicoesWeb/prop_mostrarintegra;jsessionid=2 855A1BDAAD7DEF4C17F7A7373C9E8D8.node1?codteor=142359\&filename=LegislacaoCit ada+-PL+1274/2003. Acesso em: 8 set. 2019.

BRASIL. Ministério da Saúde. Diretrizes da política nacional de saúde bucal. Brasília: Ministério da Saúde, 2004a.Disponível em: http://189.28.128.100/dab/docs/publicacoes/geral/diretrizes_ da_politica_nacional_de_saude_bucal.pdf. Acesso em: 15 set. 2019.

BRASIL. Ministério da Saúde. Secretaria de Atenção à Saúde. Departamento de Atenção Básica. Projeto SB Brasil 2003 - condiçôes de saúde bucal da população brasileira 2002-2003: resultados principais. Brasília: Ministério da Saúde, 2004b. Disponível em: http://bvsms. saude. gov.br/bvs/publicacoes/projeto_sb2004. Acesso em: 23 ago. 2019.

BRASIL. Ministério da Saúde. Secretaria de Atenção à Saúde. Secretaria de Vigilância em Saúde. SB Brasil 2010 - Pesquisa Nacional de Saúde Bucal: resultados principais. Brasilia: Ministério da Saúde, 2012. Disponível em: http:// bvsms.saude.gov.br/bvs/publicacoes/pesquisa_nacional_ saude_bucal.pdf. Acesso em: 23 ago. 2019. 
BRASIL. Ministério da Saúde. Portaria no 261, de 21 de fevereiro de 2013. Institui, no âmbito da Política Nacional de Saúde Bucal, o Programa de Melhoria do Acesso e Qualidade dos Centros de Especialidades Odontológicas (PMAQ-CEO) e o Incentivo Financeiro (PMAQCEO), denominado Componente de Qualidade da Atenção Especializada em Saúde Bucal. Diário Oficial da União. 21 de fevereiro de 2013; Seção 1.

BRASIL. Ministério da Saúde. Microdados da avaliação Externa do PMAQ CEO, 2014. Brasília: Ministério da Saúde, 2014. Disponível em: http://aps.saude.gov.br/ape/pmaq/ciclolceo/. Acesso em: 02 out. 2019.

BRASIL. Ministério da Saúde. Sala de Sala de Apoio à Gestão Estratégica, SAGE - Redes e programas: Saúde mais perto de você - Atenção Básica. Brasília: Ministério da Saúde, 2018. Disponível em: http://sage.saude.gov.br/\# . Acesso em: 23 abr. 2020.

CASOTTO, E.; CONTARATO, P.C.; FONSECA, A.B.M.; BORGES, P.K.O.; BALDANI, M.H. Atenção em Saúde Bucal no Brasil: uma análise a partir da Avaliaçáo Externa do PMAQAB. Rev Saúde Debate. v. 38, n. (spe), p. 140-157, 2014.

CHAVES, S.L.C; BRRO, S.G.; CRUZ D.N.; FIGUEIREDO, A.C.L.; MOURA, B.L.A.; CANGUSSU, M.T.C. Política Nacional de Saúde Bucal: fatores associados à integralidade do cuidado. Rev Saúde Pública. v. 44, n. 6, p. 1005-1013, 2010.

CHAVES, S.C.L.; CRUZ, D.N.; BARROS, S.G.; FIGUEIREDO, A.L. Avaliação da oferta e utilização de especialidades odontológicas em serviços públicos de atenção secundária na Bahia, Brasil. Rev Cad Saúde Pública. v. 27, n. 1, p. 143-154, 2011.

CHAVES, S.C.L.; ALMEIDA, A.M.F.L.; ROSSI, T.R.A.; SNTANA, S.F.; BARROS, S.G.; SANTOS, C.M.L. Política de Saúde Bucal no Brasil 2003-2014: cenário, propostas, açóes e resultados. Rev Ciênc Saúde Colet. v. 22, n. 6, p. 791-1803, 2017.

DUARTE, E.C.; SCHNEIDER, M.C.; PAES-SOUSA, R.; RAMALHO, W.M.; SARDINHA, L.M.V., SILVA JÚNIOR, J.B.; et al. Epidemiologia das Desigualdades em Saúde no Brasil: Um Estudo Exploratório. 1 ed. Brasília: Organização Pan-Americana da Saúde, 2002.

FACCHINI, L.A.; TOMASI, E. DILÉLIO, A.S. Qualidade da Atenção Primária à Saúde no Brasil: avanços, desafios e perspectivas. Rev Saúde Debate. v. 42, n. (spe), p. 208-223, 2018.

GOES, P.S.A.; FIGUEIREDO, N.; NEVES, J.C.; SILVEIRA, F.M.M.; COSTA J.F.R.; PUCCA JÚNIOR, G.A.; et al. Avaliação da atenção secundária em saúde bucal: uma investigação nos centros de especialidades do Brasil. Rev Cad Saúde Pública. v. 28, n. (suppl), p. 81-89, 2012.

IBGE. Pesquisa Nacional por Amostra de Domicílios: acesso e utilização de serviços de saúde 2003. Rio de Janeiro: Instituto Brasileiro de Geografia e Estatística, 2005. Disponível em: http://www.spm.gov.br/arquivos-diversos/. arquivos/integra_saude_ibge_2003. Acesso em: 29 set. 2019. 
IBGE. Pesquisa Nacional por Amostra de Domicílios - um panorama da saúde no Brasil: acesso e utilização dos serviços, condiçôes de saúde e fatores de risco e proteção à saúde. Rio de Janeiro: Instituto Brasileiro de Geografia e Estatística, 2008. Disponível em: http://biblioteca.ibge.gov. br/visualizacao/monografias/GEBIS\%20-\%20RJ/panorama.pdf. Acesso em: 29 set. 2019.

IBGE. Censo 2010. Rio de Janeiro: Instituto Brasileiro de Geografia e Estatística, 2010. Disponível em: https://censo2010.ibge.gov.br. Acesso em: 15 set. 2019.

IBGE. IBGE - cidades. Rio de Janeiro: Instituto Brasileiro de Geografia e Estatística, 2019. Disponível em http://www.ibge.gov.br/cidadesat/default.php . Acesso em: 05 mai. 2020.

INCA. Coordenação de Prevenção e Vigilância. Estimativa 2020 - incidência de câncer no Brasil. Rio de Janeiro: Instituto Nacional de Câncer José Alencar Gomes da Silva, 2019. Diponível em: https://www.inca.gov.br/sites/ufu.sti.inca.local/files//media/document//estimativa-2020incidencia-de-cancer-no-brasil.pdf. Acesso em: 07 mai. 2020.

MACHADO, F.C.A.; SILVA, J.V.; FERREIRA, M.A.F. Fatores relacionados ao desempenho de Centros de Especialidades Odontológicas. Rev Ciênc Saúde Colet. v. 20, n. 4, p. 1149-1163, 2015.

PAIM, J.; TRAVASSOS, C.; ALMEIDA, C.; BAHIA, L.; MACINKI, J. O sistema de saúde brasileiro: história, avanços e desafios. The Lancet. v. 377, n. (spe), p. 1778-1797, 2011.

PUCA JÚNIOR, G.A. A política nacional de saúde bucal como demanda social. Rev Ciênc Saúde Colet. v. 11, n. 1, p. 243- 246, 2006.

PUCCA JUNIOR, G. A. Politica Nacional de Saúde Bucal do Brasil, integralidade e acesso; o caso Brasil sorridente. 2013. 99 f. Orientadora: Nilce Santos de Melo. Tese (Doutorado em Ciências da Saúde) - Faculdade de Ciências da Saúde, Universidade de Brasília, Brasília.

SALIBA, N.A.; NAYEME, J.G.R.; MOIMAZ, S.A.S.; CECILIO, L.P.P.; GARBIN, C.A.S. Organização da demanda de um Centro de Especialidades Odontológicas. Rev Odontol da UNESP. v. 42, n. 5, p. 317-323, 2013. 


\section{Abstract}

Access and quality of secondary care and stomatology assistance in Brazil

Objective: To analyze the specialized assistance in oral health in the Centers of Dental Specialties (CEO) of Brazil, with emphasis on stomatology, in view of the increase of new cases of oral cancer in the country.

Methodology: Secondary data analysis of the 1st cycle of the CEO Access and Quality Improvement Program (PMAQ-CEO), the Brazilian Institute of Geography and Statistics (IBGE) and the Department of Primary Care of the Ministry of Health (DAB-MS). Results: Only $13.98 \%$ of the Brazilian municipalities are covered by CEO, and Stomatology is one of the most deficient specialties, present in only $65.55 \%$ of CEOs participating in PMAQ-CEO. The biopsy is performed in $82.3 \%$ of CEOs, but $60 \%$ of the services resulting from the examination are still slow and the supply of material resources is insufficient. Conclusion: The portrayal of stomatology in Brazil compromises access to diagnosis, treatment and contributes to oral diseases and morbidities, such as oral cancer.

> Keywords: Health Services Research; Secondary Care; Oral Health; Mouth Neoplasms. 